\title{
Publisher Correction to: Integrating adverse effect analysis into environmental risk assessment for exotic generalist arthropod biological control agents: a three-tiered framework
}

\author{
Débora P. Paula (1) David A. Andow - Barbara I. P. Barratt - Robert S. Pfannenstiel • \\ Philippa J. Gerard · Jacqui H. Todd • Tania Zaviezo • Maria G. Luna • \\ Claudia V. Cédola • Antoon J. M. Loomans • Andy G. Howe - Michael D. Day • \\ Clark Ehlers · Chris Green - Salvatore Arpaia - Eizi Yano • Gabor L. Lövei • \\ Norihide Hinomoto • Eliana M. G. Fontes - Carmen S. S. Pires • Pedro H. B. Togni • \\ James R. Nechols • Micky D. Eubanks • Joop C. van Lenteren
}

Published online: 18 January 2021

(C) International Organization for Biological Control (IOBC) 2020

\section{Correction to: BioControl \\ https://doi.org/10.1007/ \\ s10526-020-10053-8}

Due to typesetting errors, Table 5 was not displayed correctly in the initial online publication. The original online article has been corrected.

The original article can be found online at https://

doi.org/10.1007/s10526-020-10053-8.

D. P. Paula $(\bowtie)$ • E. M. G. Fontes · C. S. S. Pires

Department of Biological Control, Embrapa Genetic

Resources and Biotechnology, Brasília,

DF 70770-901, Brazil

e-mail: debora.pires@embrapa.br

D. A. Andow

Department of Entomology, University of Minnesota, 219

Hodson Hall, St. Paul, MN 55108, USA

B. I. P. Barratt

AgResearch, Invermay Research Centre,

PB 50034, Mosgiel, New Zealand

B. I. P. Barratt · J. H. Todd

Better Border Biosecurity, Wellington, New Zealand

R. S. Pfannenstiel

Pests, Pathogens and Biocontrol Permitting, Plant Health

Programs, USDA APHIS PPQ, 4700 River Road, Unit

133, Riverdale, MD 20737, USA
P. J. Gerard

AgResearch, Ruakura Research Centre,

PB 3123 Hamilton, New Zealand

J. H. Todd

The New Zealand Institute for Plant and Food Research Limited, Private Bag 92169, Auckland 1142, New

Zealand

T. Zaviezo

Facultad Agronomía e Ing. Forestal, Pontificia

Universidad Católica de Chile, Santiago, Chile

M. G. Luna · C. V. Cédola

CEPAVE (CONICET - UNLP), Boulevard 120 entre $60 \mathrm{y}$

64, 1900 La Plata, Argentina 
Publisher's Note Springer Nature remains neutral with regard to jurisdictional claims in published maps and institutional affiliations.

A. J. M. Loomans

National Plant Protection Organization (NPPO),

Netherlands Food and Consumer Product Safety

Authority (NVWA), Geertjesweg 15,

6706 EA Wageningen, The Netherlands

A. G. Howe

Department of Geosciences and Natural Resource Management, University of Copenhagen, Rolighedsvej

23, 1958 Frederiksberg C, Denmark

A. G. Howe

Forest Industries Research Centre, University of the Sunshine Coast, GPO Box 267, Brisbane,

QLD 4001, Australia

M. D. Day

Department of Agriculture and Fisheries,

GPO Box 267, Brisbane, QLD 4001, Australia

C. Ehlers

Environmental Protection Authority, Level 10, 215

Lambton Quay, Wellington 6011, New Zealand

C. Green

Department of Conservation,

Private Bag 68908, Newton, Auckland 1145, New

Zealand

S. Arpaia

Division Bioenergy, Biorefinery and Green Chemistry, ENEA Research Centre Trisaia, S.S. 106 Jonica km 419.5, 75026 Rotondella, MT, Italy
E. Yano

Center for Ecological Research (CER), Kyoto University, Hirano 2-509-3, Otsu, Shiga 520-2113, Japan

\section{G. L. Lövei}

Department of Agroecology, Aarhus University, Flakkebjerg Research Centre, Forsøgsvej 1, 4200 Slagelse, Denmark

N. Hinomoto

Laboratory of Ecological Information, Graduate School of Agriculture, Kyoto University, Kyoto 606-8502, Japan

P. H. B. Togni

Departamento de Ecologia, Universidade de Brasília (UnB), Campus Universitário Darcy Ribeiro, Brasília, DF 70910-900, Brazil

\section{J. R. Nechols}

Department of Entomology, Kansas State University, 123 Waters Hall, 1603 Old Claflin Place, Manhattan, KS 66506, USA

M. D. Eubanks

Department of Entomology, Texas A\&M University,

TAMU 2475, College Station, TX 77843-2475, USA

J. C. van Lenteren

Laboratory of Entomology, Department of Plant Sciences, Wageningen University and Research (WUR),

PO Box 16, 6700 AA Wageningen, The Netherlands 